\title{
Segmentation of Prostate Using Interactive Finsler Active Contours and Shape Prior
}

\author{
Foued Derraz ${ }^{1,3}$, Abdelmalik Taleb-Ahmed ${ }^{3}$, Azzeddine Chikh $^{4}$, Christina Boydev $^{3}$, \\ Laurent Peyrodie ${ }^{2}$, and Gerard Forzy ${ }^{1}$ \\ ${ }^{1}$ Faculté Libre de Médicine, Institut Catholique de Lille, France \\ ${ }^{2}$ HEI, LAGIS UMR CNRS 3304, Lille, France \\ Laurent.peyrodie@hei.fr \\ ${ }^{3}$ LAMIH UMR CNRS 8201, Le Mont Houy, 59313 Valenciennes, France \\ ${ }^{4}$ Biomedical Engineering Laboratory, Technology College, Abou Bekr Belkaid University \\ foued.derrazaicl-lille.fr, taleb@univ-valenciennes.fr, \\ az_chikh@hotmail.com, laurent.peyrodie@hei.fr, \\ gerard. forzy@ghcl. net
}

\begin{abstract}
We present a new interactive segmentation framework to segment the prostate from MR prostate imagery. We first explicitly address the segmentation problem based on fast globally Finsler Active Contours (FAC) by incorporating both statistical and geometric shape prior knowledge. In doing so, we are able to exploit the more global aspects of segmentation by incorporating user feedback in segmentation process. In addition, once the prostate shape has been segmented, a cost functional is designed to incorporate both the local image statistics as user feedback and the learned shape prior. We provide experimental results, which include several challenging clinical data sets, to highlight the algorithm's capability of robustly handling supine/prone prostate segmentation task.
\end{abstract}

Keywords: Finsler Active contours, characteristic function, shape prior, user interaction.

\section{Introduction}

Segmentation of the prostate boundary on clinical images is useful in a wide spread range of applications including calculation of prostate volume pre- and posttreatment, radiotherapy planning $[1,2,16]$, dosimetry [14] , and for creating patientspecific anatomical models [18]. Prostate volume is routinely asked as part of imaging evaluation as it helps in clinical decision making [2, 15]. However, manual segmentation of the prostate boundary is highly time-consuming and subject to interand intra-reader variability. Automatic segmentation based on deformable models such as Active Contour (AC) models have been widely used for prostate segmentation $[7,12]$ and can be split into two classes, those which fully rely on image data [9], and those which incorporate prostate prior shape information $[6,10,11]$. To deal with the complex prostate anatomy and partially missing boundaries, the shape of prostate is 
approximately assumed to be elliptical. In many cases, this segmentation is a two-part problem. First, one must properly align a set of training shapes such that any variation in shape is not due to alignment. Then, the segmentation based on deformable models can be performed under the constraint of the learned prostate shapes. However, the alignment of prostate shapes becomes increasingly difficult for a large variation in training shapes and when the training sets increase, and this is not readily allowed by existing methods. To overcome this problem, we investigated an interactive FAC model to boost the performance of segmentation results. The FAC model has been proposed as a natural way for adding directionality to the AC model [5]. This allows the AC to favor appropriate locations and suitable directions [5, 12].

In this paper, we proposed a bi-stage interactive prostate segmentation method based on fast globally active contours incorporating prior shape to segment the prostate from MR images. Then, our segmentation method based fast FAC incorporating shape prior is applied to delineate prostate boundary. User intervention is then needed to guide our method to fine-tune the final segmented shape of prostate. Finally, we apply some post-processing operations to further refine prostate boundaries. This paper organized as follows. Section 2. describes the basics of our method. In Section III, the experimental results obtained using our method are illustrated. Conclusions and future work are presented in Section 4.

\section{Segmentation Method}

The The new framework which we proposed for prostate delineation consists of two main steps: the first step is applying a FAC model incorporating prostate shape prior. The second step is refining the prostate shape by user feedback. Each of these steps is described in detail in next section.

\subsection{Finsler Active Contours in the Total Variation Framework}

We are interested in a globally interactive segmentation of an object $\Omega$ in Total Variation (TV) framework through the characteristic function [3, 9]. We proposed to segment prostate shape using a fast version of the interactive Finsler Active contours in the TV framework. We proposed a two stage fast globally Finsler Active Contours (FAC). In the first step, our fast globally FAC incorporated both statistical region, geometric shape and in the second step, the final segmentation is completed by adding an interactive user feedback. Our fast interactive segmentation can be modelled as the following energy criterion $[5,9,12]$ :

$$
\begin{aligned}
& E_{F A C}\left(\chi, \Omega, \Omega_{r e f}, \Omega_{R O I}\right)=\underbrace{\int_{\partial \Omega} k_{f}(\mathbf{x}, \partial \Omega) d a(\mathbf{x})}_{E_{\text {finsler }}(\partial \Omega)}+\lambda_{1} \underbrace{\int_{\Omega_{I}} k_{r}(\mathbf{x}, \Omega) \chi(\mathbf{x}) d \mathbf{x}}_{E_{\text {data }}(I, \Omega)} \\
& +\lambda_{2} \underbrace{\int_{\Omega_{l}} k_{s}\left(\mathbf{x}, \Omega_{\text {ref }}\right) \chi(\mathbf{x}) d \mathbf{x}}_{E_{\text {shape }}\left(\Omega, \Omega_{\text {ref }}\right)}+\lambda_{3}^{\int_{\Omega_{I}}^{\sum_{\text {user }}\left(\mathbf{x}, \Omega_{R O I}\right)} \chi(\mathbf{x}) d \mathbf{x}}
\end{aligned}
$$


Where $k_{f}$ is the anisotropic boundary descriptor, $d a(\mathbf{x})$ is surface element, $\lambda_{1}, \lambda_{2}$ and $\lambda_{3}$ are the calibration factors and the characteristic function $\chi$ framework is defined as:

$$
\chi(\mathbf{x})= \begin{cases}1 & \text { if } \mathbf{x} \in \Omega \\ 0 & \text { if } \mathbf{x} \notin \Omega\end{cases}
$$

The region descriptor $k_{r}$ is defined in the same manner as in [4], $k_{s}$ is the shape prior descriptor defined in the same manner as in [6] and $\Omega_{r e f}$ the reference prostate shape (section 2.3) and $k_{u s e r}$ the interactive user feedback descriptor which will be defined in section 2.4 .

To allow our segmentation model to detect both edge and its direction, we assume that anisotropic boundary descriptor is defined as follows:

$$
k_{f}(\mathbf{x}, \partial \Omega)=\max _{|\mathbf{p}| \leq 1, \chi}\langle\mathbf{p}, \nabla \chi(\mathbf{x})\rangle
$$

Where the potential field, $\mathbf{p}$, is defined as $\mathbf{p}=[\vec{N}, \vec{T}]$ and $\vec{N}$ is the unit inward normal vector and $\vec{T}$ the unit tangential vector (Fig. 1). This potential field allows the FAC to deform and move toward object of interest. This propriety makes the proposed segmentation method much faster since the topology of the deformed curve is more like the object to be segmented. The energy of FAC is given by:

$$
\begin{aligned}
& E_{F A C}(\chi, \mathbf{p})=\int_{\Omega} \underbrace{\max _{\mid \mathbf{p}}\langle\mathbf{p}, \nabla \chi(\mathbf{x})\rangle}_{\psi_{\mathbf{x}, \mathbf{p}}(\mathbf{x})} d a(\mathbf{x})+\lambda_{1} \int_{\Omega_{0}} k_{r}(\mathbf{x}, \Omega) \chi(\mathbf{x}) d \mathbf{x} \\
& +\lambda_{2} \int_{\Omega_{0}} k_{s}\left(\mathbf{x}, \Omega_{\text {ref }}\right) \chi(\mathbf{x}) d \mathbf{x}+\lambda_{3} \int_{\Omega_{0}} k_{\text {user }}\left(\mathbf{x}, \Omega_{\text {ROI }}\right) \chi(\mathbf{x}) d \mathbf{x}
\end{aligned}
$$

and the respective gradient descent (for $\chi$ ) and ascent (for the potential field $\mathbf{p}$ ) equations are:

$$
\left\{\begin{array}{c}
\frac{\partial \chi(\mathbf{x}, \tau)}{\partial \tau}=\operatorname{div}(\mathbf{p})-\lambda_{1} k_{r}(\mathbf{x}, \Omega)-\lambda_{2} k_{s}\left(\mathbf{x}, \Omega_{r e f}\right)-\lambda_{3} k_{\text {user }}\left(\mathbf{x}, \Omega_{R O I}\right), \chi(\mathbf{x}, \tau=0)=\mathbf{1} \\
\frac{\partial \mathbf{p}}{\partial \tau}=-\nabla \chi(\mathbf{x}, \tau)
\end{array}\right.
$$

where $\tau$ is an artificial time parameter, $\chi_{0}$ is the initialized characteristic function corresponding to the initial contour curve $\partial \Omega_{0}$. In the next section, we introduced region based term used in our fast segmentation based model. 


\subsection{Region Energy Term}

The second energy term incorporated in our Finsler AC is defined as region energy based term. We proposed in to incorporate this term as statistical Bhattachryya distance [4]. The region energy based term is usually defined as a domain integral of the region descriptor $k_{r}$ :

$$
E_{\text {data }}(I, \Omega)=\int_{\Omega_{0}} k_{r}(\mathbf{x}, \Omega) d \mathbf{x}=\sqrt{\int_{\Omega_{0}} p_{f}(I, \Omega) p_{b}(I, \Omega) d \mathbf{x}}
$$

In this study we maximize the statistical Bhattachryya distance between the foreground density probability $p_{f}(I, \Omega)$ of the object to be segmented and the background density probability $p_{b}(I, \Omega)$ such as in [12]. The region velocity $k_{r}(\mathbf{x}, \Omega)$ is expressed [10] as:

$$
k_{r}(\mathbf{x}, \Omega)=\frac{1}{2}\left\{\begin{array}{l}
\left(\frac{1}{\left|\Omega_{f}\right|}-\frac{1}{\left|\Omega_{b}\right|}\right) \sqrt{p_{f}\left(I, \Omega_{f}\right) p_{b}\left(I, \Omega_{b}\right)}+\frac{1}{\left|\Omega_{b}\right|} \int_{R^{+}} \sqrt{\frac{p_{f}\left(I, \Omega_{f}\right)}{p_{b}\left(I, \Omega_{b}\right)}} \\
\left(G_{\sigma_{\mathrm{ker}}}\left(I-I\left(\Omega_{f}\right)\right)-\sqrt{p_{b}\left(I, \Omega_{b}\right)}\right) d I \\
-\frac{1}{|\Omega|} \int_{R^{+}} \sqrt{\frac{p_{b}\left(I, \Omega_{b}\right)}{p_{f}\left(I, \Omega_{f}\right)}}\left(G_{\sigma_{\mathrm{ker}}}\left(I-I\left(\Omega_{f}\right)\right)-\sqrt{p_{f}\left(I, \Omega_{f}\right)}\right) d I
\end{array}\right\}
$$

We therefore estimate probability density by Parzen kernel, which can better describe the regions (see Fig. 1.b):

$$
p_{f / b}(I, \Omega)=\frac{1}{\left|\Omega_{f / b}\right|} \int_{\Omega_{f / b}} G_{\sigma}\left(I-I\left(\Omega_{f / b}\right)\right) d \mathbf{x}
$$

where $G_{\sigma}$ denote the Gaussian kernel and $\sigma^{2}$ the variance.

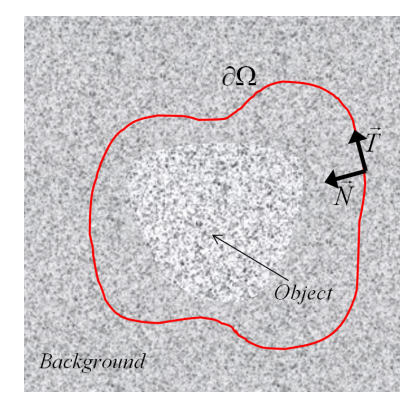

Fig. 1. Evolving FAC curve around a synthetic object guided by both normal and tangential components 


\subsection{Shape Prior Energy Term}

The shape prior descriptor is defined as the Euclidean distance between the evolving Legendre moment [6] region $\eta(\Omega)$ and the reference shapes $\left\{\Omega_{\text {ref }}^{i}, i=1, \ldots, N\right\}$ :

$$
E_{\text {shape }}\left(\Omega, \Omega_{\text {ref }}\right)=\int_{\Omega} k_{\text {shape }}\left(\mathbf{x}, \Omega_{r e f}\right) d \mathbf{x}=\sum_{p, q}^{p+q \leq N}\left|\eta_{p q}(\Omega)-\eta_{p q}\left(\Omega_{r e f}^{i}\right)\right|^{2}
$$

where the $\eta_{p q}$ are defined as follows, using the geometric moments and coefficients of the Legendre polynomials [6]. In the figure 3, the object shape is segmented using 20 learned shape prior and the segmentation is done using statistical and geometric shape prior. In the next section we introduced our interactive user term used to suitability segmentation method ( see Fig. 2).
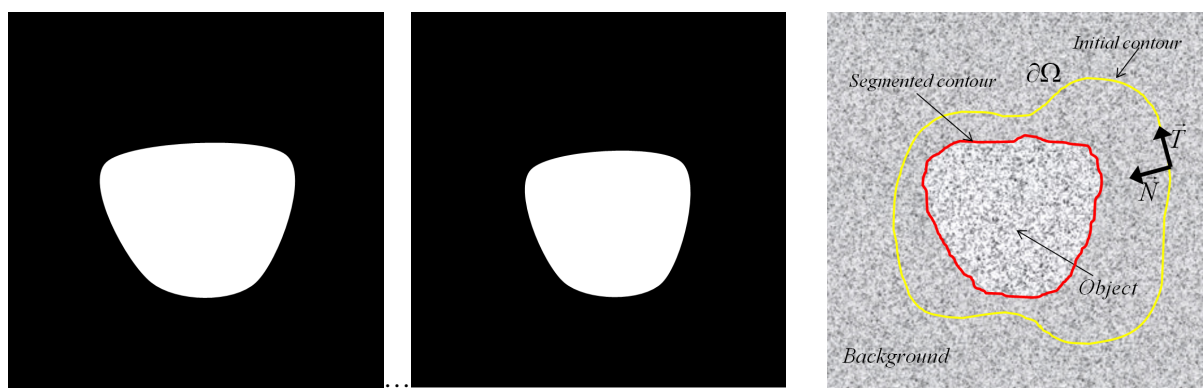

a) shapes learned as binaries surfaces b) segmentation of the object usign both statistical and geometric shape, yellow color initial contour curve and in red final segmentation done by our FAC

Fig. 2. Automatic segmentation using FAC based statistical and geometric shape prior

\subsection{User Energy Term}

Let $\mathbf{x}_{i}, i=1, \ldots, n$ denote the set of $n$ user feedback points. We define the user feedback function $L: \Omega \rightarrow \mathbb{R}$ as follows:

$$
L(\mathbf{y})=\chi(\mathbf{y})+\{1-\chi(\mathbf{y})\} \sum_{i=1}^{n} \int_{\mathbf{z} \in \Omega_{\text {ROI }}} \delta\left(\mathbf{z}-\mathbf{x}_{i}\right) d \mathbf{z}
$$

where $\delta(\mathbf{z})$ is the Dirac function and $\Omega_{R O I}$ is local region. Hence, for each $\mathbf{y} \in\left\{\mathbf{y}_{i}\right\}_{i=1}^{n}$ :

$$
L(\mathbf{y})=\left\{\begin{array}{cc}
0 & \mathbf{y} \in \Omega_{\mathbf{x}} \\
1 & \mathbf{y} \in \Omega \backslash \Omega_{\mathbf{x}} \\
\chi(\mathbf{y}) & \text { notmarked }
\end{array}\right.
$$


$L(\mathbf{x})=0$ if the feedback point is within the segmented region of the first phase and $L(\mathbf{x})=1$ if the feedback point is situated in the background. Finally, if the pixel $\mathbf{x}$ is not marked, then the indicator function is identical to indicator function $(L(\mathbf{x})=\chi(\mathbf{x}))$. (see Fig. 3.) The indicator function $L(\mathbf{x})$ is used in the formulation of the energy term which incorporates the user feedback:

$$
E_{\text {user }}\left(I, \Omega, \Omega_{R O I}\right)=\int_{\mathbf{x} \in \Omega, \Omega} \int_{\mathbf{y} \in \Omega_{R O I}}|L(\mathbf{y})-\chi(\mathbf{x})|^{2} e^{-\frac{|\mathbf{x}-\mathbf{y}|^{2}}{2 \sigma^{2}}} d \mathbf{x} d \mathbf{y}
$$

The algorithm supports two modes of user feedback. The user may either draw a cross such that its eccentricity and orientation determines the entries of the variance coefficient $\sigma$ or can provide a point-wise mouse click. The interactive energy functional $E_{\text {user }}$ is minimized, w.r.t the evolving domain $\Omega(\tau)$, is done with the shape derivative tool $[10,12]$. Thus, the user Eulerian derivative of $E_{u s e r}$ in the normal direction is as follows:

$$
\left\langle\frac{\partial E_{\text {user }}\left(I, \Omega(\tau), \Omega_{R O I}\right)}{\partial \tau}, \xi\right\rangle=\int_{\partial \Omega} k_{\text {user }}\left(\mathbf{x}, \Omega_{R O I}\right)\left\langle\xi, N_{\partial \Omega}\right\rangle d a(\mathbf{x})
$$

where the interactive velocity is expressed as:

$$
k_{\text {user }}(\mathbf{x}, \Omega)=\int_{\mathbf{y} \in \Omega_{\text {ROI }}}|L(\mathbf{y})-\chi(\mathbf{x})|^{2} e^{-\frac{|\mathbf{x}-\mathbf{y}|^{2}}{2 \sigma^{2}}} d \mathbf{y}
$$

In the figure below (Fig. 3) we present final segmentation based interactive Finsler AC in blue color superposed on the automatic segmentation based Finsler AC (red color) using statistical and geometric shape

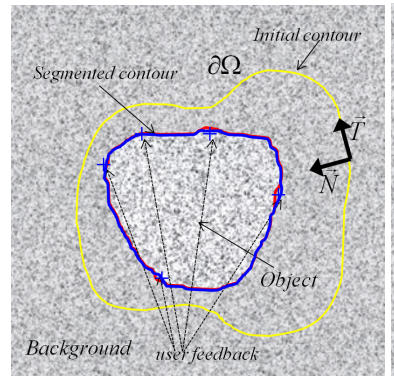

a) the selected seeds

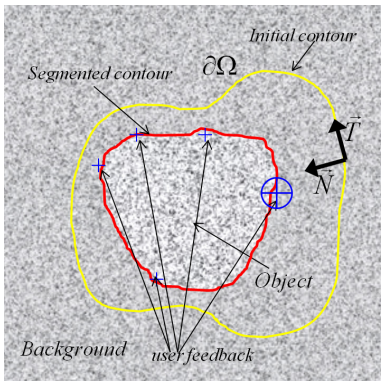

b) corrected seeds

Fig. 3. Segmentation refinement using 5 user feedback, each point selected uses 4 pixel neighbors in $\Omega_{R O I}$. 


\section{$3 \quad$ Results}

\subsection{Data and Protocol}

In this section, we provide prostate segmentation results for two data sets obtained from Saint Philibert Hospital Lille France. The MR images are pre-processed through the following pipeline: 1) spatial registration, 2) noise removal and 3) intensity standardization. We use the T1 weighted and T2 weighted MR sequences. The image sizes are $256 \times 256$ pixels, each slice thickness is $3.5 \mathrm{~mm}$ with spacing between slices of $3.9 \mathrm{~mm}$.

\subsection{Segmentation Results}

The first row shows the segmentation result by the proposed method, the second row illustrates the ground truth outlined by an expert radiologist, and the third row provides a comparison between our result and ground truth. We observe that our segmentation result is sufficiently close to the result provided by a radiologist. In addition to visual evaluation, we use Dice measure (DSC) to quantitatively evaluate the segmentation result. The Dice measure is defined as:

$$
\operatorname{DSC}(A, B)=2 \frac{|A \cap B|}{|A|+|B|}
$$

where $A$ is the segmentation result, $B$ is the ground truth provided by an expert radiologist, and $\mid \cdot$ denotes the number of segmented pixels.

To quantify the accuracy of the segmentation, we measured the Dice Similarity Coefficient (DSC) between the manually segmented prostate and our segmentation method. We provide not only qualitative results, but also give quantitative results in the form of the DSC to illustrate the viability of the proposed method in the context of prostate segmentation(see Fig 4.).

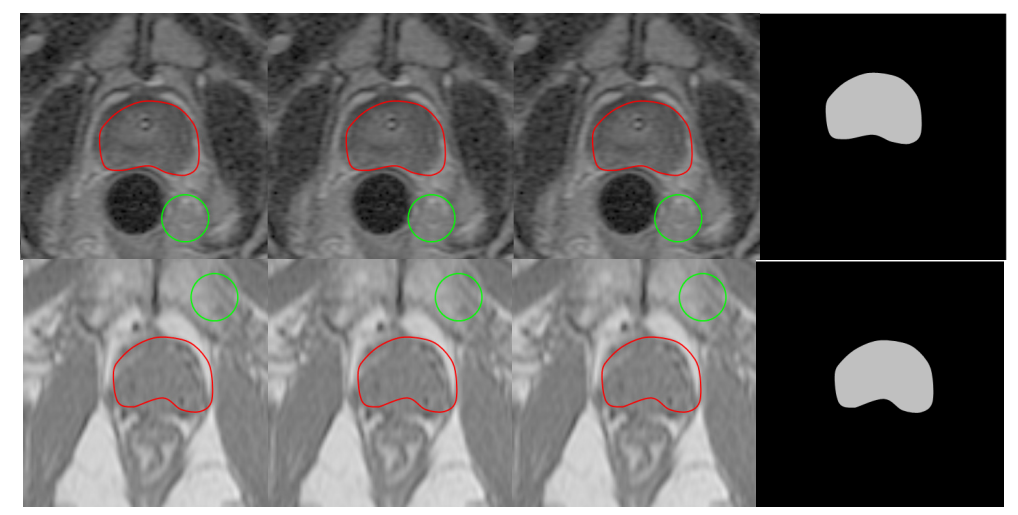

Fig. 4. Segmentation by TV FAC. In yellow color traditional FAC, red color segmentation results of our method. The DSC for the first image 85,63\% and the second image of 82,69\%. 


\section{Conclusion}

This paper has presented an interactive prostate segmentation MR images method based active contours. The segmentation is achieved in two stages. In the first stage, the patient prostate is segmented using a fast globally FAC incorporating statistical and shape prior knowledge. The position and orientation are dependent on prior for the boundary segmentation in Finsler metrics. Finsler active contours provide an alternative approach to integrating image-based priors on the location and orientation of the traditional boundary descriptor. Future work will address extending other classes of energies that can be optimized in TV framework.

\section{References}

[1] Zwiggelaar, R., Zhu, Y., Williams, S.: Semi-automatic Segmentation of the Prostate. In: Perales, F.J., Campilho, A.C., Pérez, N., Sanfeliu, A. (eds.) IbPRIA 2003. LNCS, vol. 2652, pp. 1108-1116. Springer, Heidelberg (2003)

[2] Villeirs, G., De Meerleer, G.: Magnetic resonance imaging (MRI) anatomy of the prostate and application of MRI in radiotherapy planning. Eur. J. Radiol. 63(3), 361-368 (2007)

[3] Bresson, X., Esedoglu, S., Vandergheynst, P., Thiran, J., Osher, S.: Fast Global Minimization of the Active Contour/Snake Model. JMIV 28(2) (2007)

[4] Michailovich, O., Rathi, Y., Tannenbaum, A.: Image Segmentation Using Active Contours Driven by the Bhattacharyya Gradient Flow. IEEE Trans. IP 16(11), 2787-2801 (2007)

[5] Melonakos, J., Pichon, E., Angenent, S., Tannenbaum, A.: Finsler active contours. IEEE Trans. PAMI 30(3), 412-423 (2008)

[6] Foulonneau, A., Charbonnier, P., Heitz, F.: Affine-Invariant Geometric Shape Priors for Region-Based Active Contours. IEEE Trans. PAMI 28(8), 1352-1357 (2006)

[7] Pasquier, D., Lacornerie, T., Vermandel, M., Rousseau, J., Lartigau, E., Betrouni, N.: Automatic Segmentation of Pelvic Structures From Magnetic Resonance Images for Prostate Cancer Radiotherapy. Int. Jnl. of Radiation Oncology, Biology, Physics 68(2), 592-600 (2007)

[8] Mahdavi, S., Chng, N., Spadinger, I., Morris, W.J., Salcudean, S.E.: Semi-automatic segmentation for prostate interventions. Medical Image Analysis 15(2), 226-237 (2011)

[9] Derraz, F.: Optimal segmentation by fast binary geometric active contours, PhD Thesis (2010)

[10] Duay, V., Houhou, N., Thiran, J.P.: Atlas-based segmentation of medical images locally constrained by level sets. In: IEEE ICIP 2005, vol. 2, pp. 1286-1289 (2005)

[11] Martin, S., Daanen, V., Troccaz, J.: Atlas-based prostate segmentation using an hybrid registration. Int. J. CARS 3, 485-492 (2008)

[12] Aubert, G., Barlaud, M., Faugeras, O., Jehan-Besson, S.: Image segmentation using active contours: Calculus of variations or shape gradients? SIAM Applied Mathematics 63 (2002)

[13] Klein, S., Staring, M., Pluim, J.P.W.: Evaluation of optimization methods for nonrigid medical image registration using mutual information and B-splines. IEEE Trans. Image Process. 16(12), 2879-2890 (2007) 
[14] Pasquier, D., Peyrodie, L., Denis, F., Pointreau, Y., Bera, G., Lartigau, E.: Segmentation automatique des images pour la planification dosimetrique en radiotherapie. Cancer/Radiotherapie 14(S.1), 6-13 (2010)

[15] Vikal, S., Haker, S., Tempany, C., Fichtinger, G.: Prostate contouring in MRI guided biopsy. In: SPIE Conf., vol. 7259, p. 144 (2009)

[16] Pasquier, D., Lacornerie, T., Vermandel, M., Rousseau, J., Lartigau, E., Betrouni, N.: Automatic segmentation of pelvic structures from magnetic resonance images for prostate cancer radiotherapy. Int. J. Radiat. Oncol., Biol., Phys. 68(2), 592-600 (2007)

[17] Liu, X., Langer, D.L., Haider, M.A., Van der Kwast, T.H., Evans, A.J., Wernick, M.N., Yetik, I.S.: Unsupervised Segmentation of the Prostate Using MR Images Based on Level Set with a Shape Prior. In: IEEE EMBC 2009 (2009) 